(C)1983. The Genetical Society of Great Britain

\title{
DETECTION OF FREQUENCY-DEPENDENT SELECTION IN SEQUENCES OF DATA
}

\author{
L. M. COOK \\ Department of Zoology, University of Manchester, Manchester M13 9PL, U.K.
}

Received 14.ii.83

The cross-product ratio $w_{i}=p_{i} q_{i+1} / q_{i} p_{i+1}$ may be used as a measure of selection. The values $p$ and $q$ may be numbers in successive generations of two morphs or of two species, or they may be the numbers of two forms falling into two classes. For example, instead of successive generations they may be mated individuals versus unmated or those affected versus unaffected by some sort of disease. The quantities in the two categories are often, but not necessarily, expressed as frequencies, so that $p=1-q$.

It is often of interest to know whether $w_{i}$ varies with $q_{i}$. For an infinite population $w$ is independent of $q$, so that for independent sets of data the regression of $w$ on $q$ will be zero if the selection measured by $w$ is frequency-independent, and positive or negative if it is frequency-dependent. A negative covariance (or regression or correlation coefficient) for $w$ or $\log w$ on $q$ indicates negative frequency dependence. Attention has recently been drawn to the fact that finite sample size leads to bias which may invalidate such tests (Goux and Anxolabéhère, 1980; Anxolabéhère et al., 1982). It is also worth pointing out that the method is invalidated if the data available are sequences of frequencies and $w$ is calculated for successive pairs.

The shortest sequence which could be used to provide an indication of the relation is three successive pairs of frequencies. These allow $w_{0}$ and $w_{1}$ to be calculated. The slope of $w$ on $q$ is $\left(w_{0}-w_{1}\right) /\left(q_{0}-q_{1}\right)$. Suppose that selection is constant, so that $w_{0}$ and $w_{1}$ have the same mean (independent of $q$ ) but vary randomly about it. If $w_{i}=1-s_{i}$ then $q_{1}=w_{0} q_{0} /\left(1-q_{0}+w_{0} q_{0}\right)=\left(1-s_{0}\right) q_{0} /\left(1-s_{0} q_{0}\right)$, and the slope is $\left(s_{1}-s_{0}\right)\left(1-s_{0} q_{0}\right) / s_{0} q_{0}\left(1-q_{0}\right)$. This expression is negative if $\left(s_{1}-s_{0}\right) / s_{0}<0$. When the mean for $s$ is zero that will be the case in the circumstances shown below.

\begin{tabular}{c|cc} 
& \multicolumn{2}{|c}{$s_{0} s_{1}$} \\
$s_{0} / s_{1}$ & $>0$ & $<0$ \\
\hline$>1$ & - & - \\
$<1$ & + & -
\end{tabular}

When $q$ is near $0 \cdot 5$, random fluctuations in $s$ will be distributed symmetrically, so that each outcome is equally likely, and the probability of a negative slope by chance in the absence of selection is 0.75 , not 0.5 .

Stochastic simulations made under a range of conditions have indicated that for longer sequences the correlation or regression coefficients will usually be totally misleading as indicators of frequency-dependent selection in sequences of data. A solution would be to ignore every third frequency 
and to calculate the coefficient from the remaining pairs. The auto-correlation effect is then avoided and one is left only with the difficulties discussed by Goux and his colleagues.

I am grateful to Dr Wallace Arthur for discussion of this problem.

\section{REFERENCES}

GOUX, J. M. AND ANXOLABEHERE, D. 1980. The measurement of sexual isolation and selection: a critique. Heredity, 45, 255-262.

ANXOLA BEHERE, D., GOUX, J. M. AND PERIQUET, G. 1982. A bias in estimation of viabilities from competition experiments. Heredity, 48, 271-282. 